\title{
Linearly polarized single photon antibunching from a site-controlled InGaN quantum dot
}

\author{
Tomas Jemsson, Houssaine Machhadani, Fredrik K Karlsson, Chih-Wei Hsu and Per-Olof \\ Holtz
}

\section{Linköping University Post Print}

\section{Tweet}

N.B.: When citing this work, cite the original article.

Original Publication:

Tomas Jemsson, Houssaine Machhadani, Fredrik K Karlsson, Chih-Wei Hsu and Per-Olof Holtz, Linearly polarized single photon antibunching from a site-controlled InGaN quantum dot, 2014, Applied Physics Letters, (105), 8, 081901.

http://dx.doi.org/10.1063/1.4893476

Copyright: American Institute of Physics (AIP) http://www.aip.org/

Postprint available at: Linköping University Electronic Press http://urn.kb.se/resolve?urn=urn:nbn:se:liu:diva-112065 


\section{AIP |Applied Physics Letters}

\section{Linearly polarized single photon antibunching from a site-controlled InGaN quantum}

dot

Tomas Jemsson, Houssaine Machhadani, K. Fredrik Karlsson, Chih-Wei Hsu, and Per-Olof Holtz

Citation: Applied Physics Letters 105, 081901 (2014); doi: 10.1063/1.4893476

View online: http://dx.doi.org/10.1063/1.4893476

View Table of Contents: http://scitation.aip.org/content/aip/journal/apl/105/8?ver=pdfcov

Published by the AIP Publishing

\section{Articles you may be interested in}

Observations of Rabi oscillations in a non-polar InGaN quantum dot

Appl. Phys. Lett. 104, 263108 (2014); 10.1063/1.4886961

Single photon emission from site-controlled InGaN/GaN quantum dots

Appl. Phys. Lett. 103, 192114 (2013); 10.1063/1.4830000

Single photon emission from InGaN/GaN quantum dots up to $50 \mathrm{~K}$

Appl. Phys. Lett. 100, 061115 (2012); 10.1063/1.3683521

Room-temperature quantum-dot-like luminescence from site-controlled InGaN quantum disks

Appl. Phys. Lett. 99, 263105 (2011); 10.1063/1.3672441

Effects of piezoelectricity and spontaneous polarization on localized excitons in self-formed InGaN quantum dots J. Appl. Phys. 94, 407 (2003); 10.1063/1.1576490

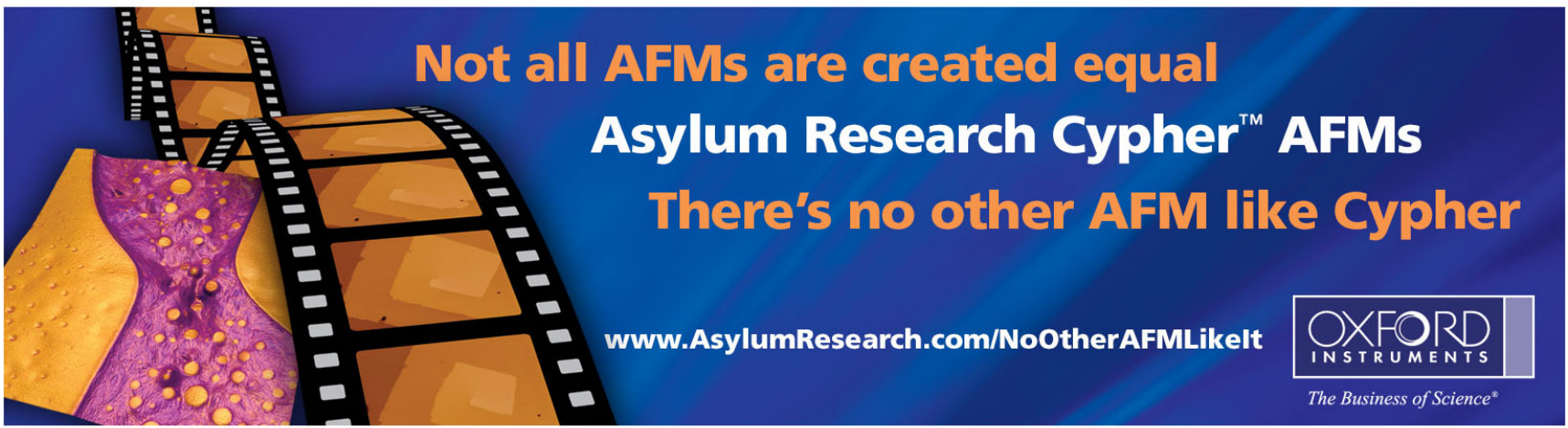




\title{
Linearly polarized single photon antibunching from a site-controlled InGaN quantum dot
}

\author{
Tomas Jemsson, Houssaine Machhadani, K. Fredrik Karlsson, Chih-Wei Hsu, \\ and Per-Olof Holtz \\ Department of Physics, Chemistry, and Biology (IFM), Semiconductor Materials, Linköping University, \\ S-58183 Linköping, Sweden
}

(Received 1 May 2014; accepted 2 June 2014; published online 25 August 2014)

\begin{abstract}
We report on the observation of linearly polarized single photon antibunching in the excitonic emission from a site-controlled InGaN quantum dot. The measured second order coherence function exhibits a significant dip at zero time difference, corresponding to $g_{m}^{2}(0)=0.90$ under continuous laser excitation. This relatively high value of $g_{m}^{2}(0)$ is well understood by a model as the combination of short exciton life time ( $320 \mathrm{ps})$, limited experimental timing resolution and the presence of an uncorrelated broadband background emission from the sample. Our result provides the first rigorous evidence of InGaN quantum dot formation on hexagonal GaN pyramids, and it highlights a great potential in these dots as fast polarized single photon emitters if the background emission can be eliminated. (C) 2014 AIP Publishing LLC. [http://dx.doi.org/10.1063/1.4893476]
\end{abstract}

Semiconducting quantum dots (QDs) are getting increasingly more attention as light sources for future quantum information applications (QIA). ${ }^{1}$ These applications require sources of single or quantum correlated photons. In addition, it is desired that the photon emitters are sitecontrolled and that they are operating close to room temperature. Some QIA, such as quantum key distribution with the BB84 protocol, can be realised with linearly polarized photons, where the polarization has to be switched on a photonby-photon level. ${ }^{2}$

QDs are small inclusions in a semiconductor matrix with band offsets that provide trapping and quantum confinement of the charge carriers. A quantum dot therefore exhibits a discrete set of atom-like energy states, and the optically active transitions between these states give rise to a spectrum of sharp and well-defined emission lines. A single photon emitter can then be produced by filtering out all except one emission line from an individual quantum dot. This was first realised experimentally in year 2000 using conventional Stranski-Krastanov (SK) grown InAs/GaAs quantum dots. ${ }^{3}$ To obtain an emission of strongly linearly polarized photons in this material system, complicated micro cavity structures with a polarized optical mode in resonance with a QD emission line are needed. ${ }^{4,5}$

Highly linearly polarized emission has been reported for various bare QDs based on Al,In,Ga-nitrides, including SK grown QDs, ${ }^{6} \mathrm{QDs}$ etched out from a planar single quantum well $(\mathrm{QW}){ }^{7}$ and QDs formed in nanorods. ${ }^{8}$ However, none of these studies reports any controlled or preferential polarization direction. Recently, the small split-off energy of the nitrides was identified as the main cause of the high degree of polarization for asymmetric quantum dots. ${ }^{9}$ The nitrides are thus more suitable for the generation of polarized photons emission than other materials such as InAs and GaAs with large split-off energy.

Another attractive property of the nitrides is the wide tunability of the band gaps with the potential to emit photons in a vast energy range from infra-red to deep ultra-violet, ${ }^{10}$ and the possibility to achieve the deep confinement potential needed for high temperature operation. Single SK grown GaN/AlN QDs have been demonstrated to emit single photons up to $200 \mathrm{~K},{ }^{11}$ and recently, single photon emission up to room temperature was reported for a site-controlled $\mathrm{GaN}$ / AIN QD formed on the tip of a nanorod. ${ }^{12}$

A promising approach for fabrication of nitride-based QDs is to deposit a thin layer of InGaN on top of hexagonal GaN micro pyramids formed by selective area growth. ${ }^{13}$ Pyramids grown with this approach have been shown to exhibit single and sharp InGaN related emission lines with high degree of linear polarization, indicating the formation of one asymmetric site-controlled InGaN quantum dot on each pyramid. ${ }^{14}$ Moreover, a simple elongation of the pyramid base gives control of the polarization direction of the InGaN emission, as it is found to be well-aligned with the designed elongation. ${ }^{15}$ The first experimental evidence of a trion in a nitride QD has also been reported for these structures. ${ }^{16}$ However, although several indirect evidences of QD formation in these pyramidal structures have been observed, no direct proof has yet been provided.

In this Letter, we report on time-correlated single photon spectroscopy (TCSPS) of the InGaN-related emission from pyramidal structures. TCSPS tests the single photon emitting properties, and it can therefore provide the ultimate proof of a QD emission. We observe a clear photon-antibunching from a site controlled InGaN QD, with the measured second order coherence function at zero delay time $g_{m}^{2}(0)=0.90$ under continuous wave excitation. This relatively large value of $g_{m}^{2}(0)$ is highly affected by an uncorrelated background emission as well as by the short exciton lifetime compared to the timing resolution of the measurement setup, as is shown with a theoretical model.

The structure used in this investigation is grown by a horizontal low pressure hot-wall metal-organic chemical vapor deposition system. A $\sim 100 \mathrm{~nm}$ AlN nucleation layer, followed by a $2 \mu \mathrm{m} \mathrm{GaN}$ layer, is deposited on a Si-face onaxis $4 \mathrm{H}-\mathrm{SiC}$ substrate. Selective area regrowth of $\mathrm{GaN}$ 
pyramids is performed in the lithographically patterned $\mathrm{SiN}$ masked GaN/AlN/SiC template. ${ }^{14}$ (See the inset in Fig. 1) The pyramids are covered by a thin layer of $\mathrm{InGaN}$ forming nanoscopic 3D islands in a SK-like growth mode on the truncated (0001) top surface. The structure is then covered by a layer of $\mathrm{GaN}$ to finalize the formation of QD inclusions in the tip of the pyramids. ${ }^{13}$ The pyramids have a base diameter of $3 \mu \mathrm{m}$, and they are ordered in square $21 \times 21$ arrays with $5.5 \mu \mathrm{m}$ pitch.

A $355 \mathrm{~nm}$ continuous wave (cw) diode pumped solid state laser was used for the microphotoluminescence $(\mu \mathrm{PL})$ measurements. The excitation laser was led through a refractive microscope objective $(\mathrm{NA}=0.42$ ) and focused down to a spot size of about $1 \mu \mathrm{m}$ on the sample in a continuous flow helium cryostat, keeping the temperature at $25 \mathrm{~K}$ or lower. The photons emitted from the sample were collected by the same objective and guided to a monochromator with a focal length of $550 \mathrm{~mm}$. The luminescence spectra were recorded by a charge coupled device (CCD), and the resolution of the setup was $0.28 \mathrm{meV}$ for the relevant energy range.

TCSPS measurements were made with both cw and pulsed laser excitation. Compared to $\mu \mathrm{PL}$ measurements, only the signal side was altered for the $\mathrm{cw}$ measurements, with the light guided to a Hanbury-Brown and Twiss setup. ${ }^{17}$ A 50/50 beam splitter directed the photons in each leg through a monochromator to an avalanche photo diode (APD). ${ }^{18}$ Time differences between subsequent photons were recorded by a TCSPC module with an overall instrument time constant $\tau_{i}=0.7 \mathrm{~ns}$, and histograms were generated with the bin-width set to $\tau_{B W}=512 \mathrm{ps}$. For the pulsed excitation, a frequency tripled Ti-sapphire laser generated ps excitation pulses at $266 \mathrm{~nm}$ wavelength, and a reflective objective $(\mathrm{NA}=0.5)$ was used to focus the light to a spot size of $\sim 2 \mu \mathrm{m}$ and to collect the luminescence. In all TCSPC measurements, care was taken to use a low laser intensity, being well below the saturation of the exciton state, not to

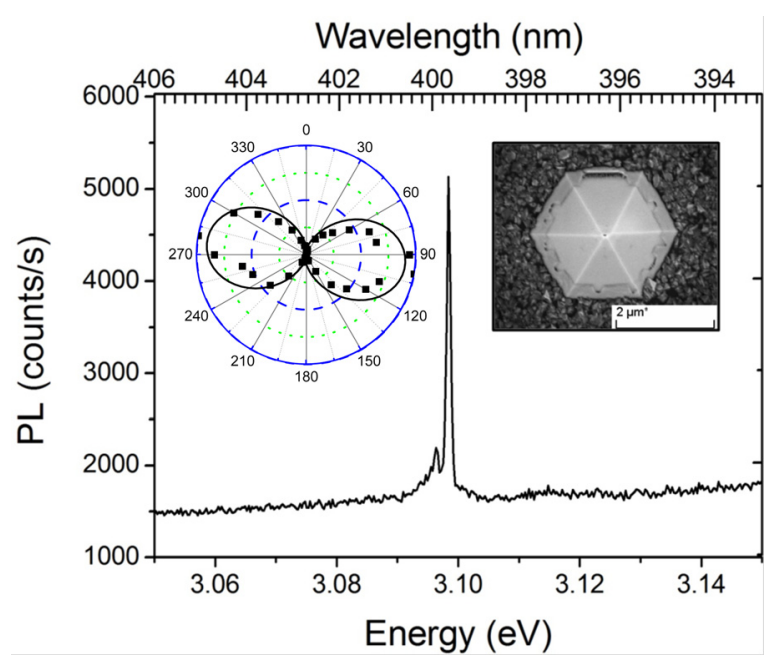

FIG. 1. $\mu \mathrm{PL}$ spectrum of the QD recorded with a CCD. The trion is seen on the low energy side of the exciton. Left inset: Measured polarization dependence of the exciton (squares). The degree of polarization is $\gtrsim 96 \%$. The solid line fitting is of the form $I(\theta)=I_{\min } \cdot \sin ^{2}(\theta-\varphi)+I_{\max } \cdot \cos ^{2}(\theta-\varphi)$, where $I_{\min }=0, I_{\max }=0.75$, and $\varphi=93^{\circ}$. The green dotted concentric circles have radius 0.25 and 0.75 . Right inset: A SEM top view of one pyramid. alter the temporal profile of the antibunching dip in the correlation histogram. ${ }^{19}$

A photon counter was also used to record luminescence spectra with the APDs. These measurements give a precise information about the intensities of the broadband background emission $(B)$ as well as the narrow linewidth QD signal $(S)$ during the actual TCSPS experiments. A relative signal $\rho=\frac{S}{S+B}=0.65 \pm 0.05$ was obtained with $\mathrm{cw}$ excitation, while a lower value $\rho=0.45$ was obtained for the pulsed excitation.

The $\mu \mathrm{PL}$ spectrum of the measured QD acquired at low excitation power is dominated by the emission of the single exciton (see Fig. 1). A weaker spectral feature originating from the negative trion, is observed $2 \mathrm{meV}$ below the predominant exciton in the low power spectrum, but this feature gains intensity and becomes comparable with the exciton intensity at higher power. ${ }^{16}$ The lifetime of the exciton has been estimated to be $\tau_{x}=320 \mathrm{ps}$, determined in a similar way as in Ref. 16. The exciton peak has a high degree of linear polarization $\gtrsim 96 \%$ (see the inset of Fig. 1), which is a value typical for most QDs measured in this sample.

TCSPS data of the exciton emission are shown in Fig. 2. The data are the result of several merged measurements in order to reduce the statistical fluctuations, corresponding to a total acquisition time of more than $14 \mathrm{~h}$. The large number of coincidence counts in the data provides significance to the measured anti bunching dip at a value of $g_{m}^{2}(0)=0.90$.

The corresponding value of $g_{m}^{2}(0) \approx 0.8$ obtained under pulsed $266 \mathrm{~nm}$ excitation has a greater uncertainty due to a smaller number of coincidences. However, by analysing a more extended time span of the $g_{m}^{2}(\tau)$ histogram, we can estimate that the probability for the dip being just a statistical fluctuation is less than $1 \%$.

The measured antibunching dip evidences a nonclassical nature of the light emitted from the sample which ultimately proves the existence of a $\mathrm{QD}$ on the GaN pyramidal structure. However, a QD is ideally a single photon emitter with $g^{2}(0)=0$. Consequently, one would expect a much more pronounced dip, $g_{m}^{2}(0) \approx 0$, in the measured coherence function.

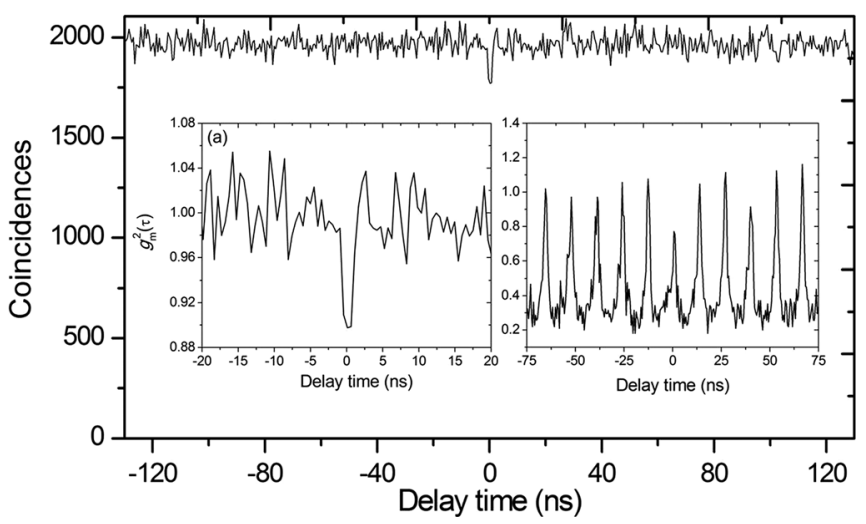

FIG. 2. Auto correlation measurement of the exciton excited with a continuous wave laser. The diagram comprises of $10^{6}$ coincidences. Left inset: The normalized $g_{m}^{2}(\tau)$ around $\tau=0$ blown up. Right inset: Auto correlation measurement with pulsed laser, resulting in $g_{m}^{2}(0) \approx 0.8$, based on approximately 12000 coincidences. 
There are three parameters that strongly affect the measured $g_{m}^{2}(0)$ value of a single photon emitter under continuous excitation: (i) The bin width $\tau_{B W}$ of the histogram, (ii) the timing resolution $\tau_{i}$ of the instrumental setup, and (iii) the relative intensity $\rho$ of the single photon source with respect to the total intensity detected, including uncorrelated background light. An expression of the actual second order coherence function $g^{2}(\tau)$ corrected for superimposed uncorrelated background emission was provided in Ref. 20 under the assumption that the feeding of the QD is much slower than the exciton lifetime $\tau_{x}$,

$$
g^{2}(\tau)=1-\rho^{2} e^{-|\tau| / \tau_{X}},
$$

where $\tau$ is the time difference between the detected pairs of photons. An expression of the measured second order coherence function $g_{m}^{2}(\tau)$ is obtained by convoluting $g^{2}(\tau)$ with the instrument temporal response function

$$
g_{m}^{2}(\tau)=\frac{1}{2 \tau_{i}} \int_{-\infty}^{+\infty} g^{2}(\tau) e^{-|\tau-t| / \tau_{i}} d t
$$

Finally, the measured value at zero delay time, $g_{m}^{2}(0)$, is given by integration of $g_{m}^{2}(\tau)$ in a symmetric time span around $\tau=0$ of total width $\tau_{B W}$

$$
g_{m}^{2}(0)=\frac{1}{\tau_{B W}} \int_{-\tau_{B W} / 2}^{+\tau_{B W} / 2} g_{m}^{2}(\tau) d \tau .
$$

Equation (3) gives the possibility to analyse the influence of $\tau_{B W}, \tau_{i}$, and $\rho$ on the measured value $g_{m}^{2}(0)$. We start this analysis by assuming an ideal experimental setup with $\tau_{i}=0$ ns, i.e. a setup with zero uncertainty in the measured time differences. An ideal QD based single photon emitter ( $\rho=1)$ with the actual exciton life time $\tau_{x}=320 \mathrm{ps}$ and the bin width $\tau_{B W}=512$ ps brings the value of $g_{m}^{2}(0)$ from nominally zero up to $g_{m}^{2}(0)=0.31$. An even less pronounced dip in the coherence function is expected for the ideal single photon emitter with a realistic instrument timing resolution $\tau_{i}=700 \mathrm{ps}$, which gives $g_{m}^{2}(0)=0.70$. Since $\tau_{x}$ is much shorter than $\tau_{i}$, a narrowing and closing of the dip in the histogram takes place around zero time difference. From the PL spectra, it is obvious that the QD emission is overlapping with a spectral background, which reduces the relative intensity of the QD from unity to $\rho=0.65$, yielding an expected value of the second order coherence function $g_{m}^{2}(0)=0.87$. This value is indeed close to the measured value $g_{m}^{2}(0)=0.90$. Thus, we can explain the absence of a pronounced dip as the combined effect of a short exciton life time in comparison with the experimental timing resolution and bin width, and the fact that the QD emission is spectrally overlapping with uncorrelated background emission. In fact, these results indicate that the QD itself seems to be a close to ideal single photon source since the measured high value for $g_{m}^{2}(0)$ is explained entirely by extrinsic effects.

Figure 3 shows the computed value of $g_{m}^{2}(0)$ versus the relative signal intensity $\rho$ for some relative values of $\tau_{i}$ and $\tau_{B W}$. Deduced values from our experiment are $\rho=0.65$, $\tau_{B W} / \tau_{x}=512 / 320=1.6$ and $\tau_{i} / \tau_{x}=700 / 320 \approx 2.2$. It is clear from the two top curves in Fig. 3 that $g_{m}^{2}(0)$ obtained

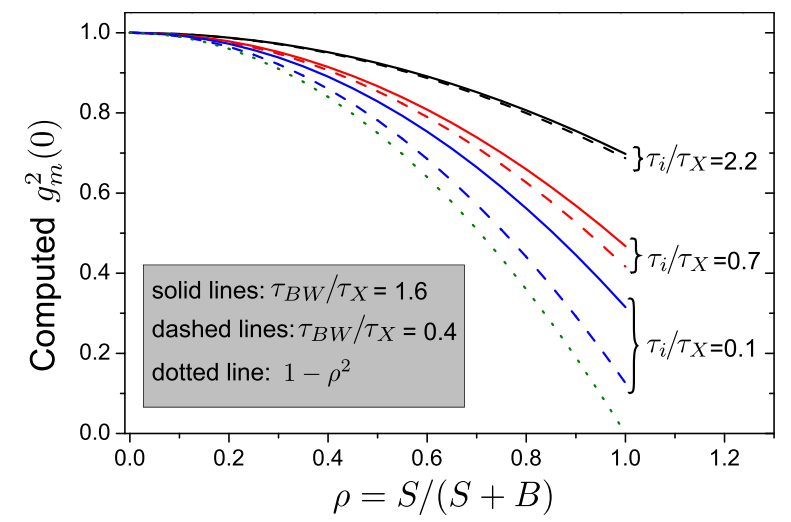

FIG. 3. The outcome of the calculations of $g_{m}^{2}(0)$ as a function of $\rho$ for different values of $\tau_{i} / \tau_{x}$ and $\tau_{B W} / \tau_{x}$ for the case of cw excitation. A plot of $1-\rho^{2}$ is added for comparison with measurements employing pulsed excitation.

under the current conditions is essentially unaffected by any reduction of the histogram bin width. This is always true unless $\tau_{i} / \tau_{x} \ll 1$. Another general result is that if $\rho<0.3$, the uncorrelated background is sufficiently strong to cause $g_{m}^{2}(0) \geq 0.9$, even under the best instrumental conditions.

By using pulsed excitation with a pulse repetition time much longer than $\tau_{i}, \tau_{B W}$, and also $\tau_{x}$, ${ }^{21}$ the influence of these three parameters on $g_{m}^{2}(0)$ can be completely avoided. In this case, the value of $g_{m}^{2}(0)$ is determined solely by the relative signal intensity $\rho$ according to $g^{2}(0)=1-\rho^{2}$. Thus, the expected value $g_{m}^{2}(0)$ for our experimental conditions with $\rho=0.46$ is $g_{m}^{2}(0)=0.79$, which should be compared with the actual measured value of $g_{m}^{2}(0) \approx 0.8$. Again, the good agreement between the expected and the measured values of $g_{m}^{2}(0)$ indicates that the QD itself is a good single photon source.

The analysis above indicates that the InGaN/GaN pyramidal QD system is promising as a rapid polarized single photon emitter, but the spectrally overlapping background emission must be significantly reduced for any application. Most of this background originates from a broadband emission around the bottom edges of the hexagonal pyramid. ${ }^{15}$ Thus, one way to improve the single photon characteristics would be to add an extra post-growth processing step, where the bottom edges are either etched away or optically screened by a metal film.

In summary, we have observed a clear photon anti bunching with $g_{m}^{2}(0)=0.90$ in the linearly polarized emission from a site controlled InGaN QD on top of a GaN truncated pyramid. This is the first direct evidence of InGaN QD formation on hexagonal GaN pyramids. A model was introduced to analyse the deterioration of the second order coherence function at zero delay times. Within this model, the relatively high value measured for $g_{m}^{2}(0)$ could be well understood as the effects of low experimental timing resolution in combination with a short exciton life time and the existence of an uncorrelated background emission. These results indicate that the intrinsic properties of the InGaN QD are promising for applications as a fast and polarized single photon emitter, but the background emission from the pyramidal structure has to be significantly reduced in order to achieve useful single photon characteristics. 
The authors H.M., K.F.K., and P.O.H. acknowledge financial support from the Carl Trygger Foundation for Scientific Research, the Swedish Research Council (VR), the Nano-N consortium funded by the Swedish Foundation for Strategic Research (SSF), and the Knut and Alice Wallenberg Foundation. We acknowledge support from the Swedish Government Strategic Research Area in Materials Science on Functional Materials at Linköping University (Faculty Grant SFO-Mat-LiU \# 2009-00971). T.J. gratefully acknowledges financial support from the Font-D, at Linköping University.

${ }^{1}$ M. D. Eisaman, J. Fan, A. Migdall, and S. V. Polyakov, Rev. Sci. Instrum. 82, 071101 (2011).

${ }^{2}$ N. Gisin, G. Ribordy, W. Tittel, and H. Zbinden, Rev. Mod. Phys. 74, 145 (2002).

${ }^{3}$ P. Michler, A. Kiraz, C. Becher, W. V. Schoenfeld, P. M. Petroff, L. Zhang, E. Hu, and A. Imamoglu, Science 290, 2282 (2000).

${ }^{4}$ M. T. Rakher, N. G. Stoltz, L. A. Coldren, P. M. Petroff, and D. Bouwmeester, Appl. Phys. Lett. 93, 091118 (2008).

${ }^{5}$ D. C. Unitt, A. J. Bennett, P. Atkinson, D. A. Ritchie, and A. J. Shields, Phys. Rev. B 72, 033318 (2005).

${ }^{6}$ S. Kremling, C. Tessarek, H. Dartsch, S. Figge, S. Hoefling, L. Worschech, C. Kruse, D. Hommel, and A. Forchel, Appl. Phys. Lett. 100, 061115 (2012).
${ }^{7}$ L. Zhang, C.-H. Teng, T. A. Hill, L.-K. Lee, P.-C. Ku, and H. Deng, Appl. Phys. Lett. 103, 192114 (2013).

${ }^{8}$ S. Deshpande and P. Bhattacharya, Appl. Phys. Lett. 103, 241117 (2013).

${ }^{9}$ S. Amloy, K. F. Karlsson, and P. O. Holtz, e-print arXiv:1311.5731.

${ }^{10}$ N. Grandjean and M. Ilegems, Proc. IEEE 95, 1853 (2007).

${ }^{11}$ S. Kako, C. Santori, K. Hoshino, S. Goetzinger, Y. Yamamoto, and Y. Arakawa, Nat. Mater. 5, 887 (2006).

${ }^{12}$ M. J. Holmes, K. Choi, S. Kako, M. Arita, and Y. Arakawa, Nano Lett. 14, 982 (2014).

${ }^{13}$ A. Lundskog, J. Palisaitis, C. W. Hsu, M. Eriksson, K. F. Karlsson, L. Hultman, P. O. A. Persson, U. Forsberg, P. O. Holtz, and E. Janzen, Nanotechnology 23, 305708 (2012).

${ }^{14}$ C.-W. Hsu, A. Lundskog, K. F. Karlsson, U. Forsberg, E. Janzen, and P. O. Holtz, Nano Lett. 11, 2415 (2011).

${ }^{15}$ A. Lundskog, C.-W. Hsu, K. F. Karlsson, A. Supaluck, D. Nilsson, U. Forsberg, P. O. Holtz, and E. Janzn, Light: Sci. Appl. 3, e139 (2014).

${ }^{16}$ C.-W. Hsu, E. S. Moskalenko, M. O. Eriksson, A. Lundskog, K. F. Karlsson, U. Forsberg, E. Janzen, and P. O. Holtz, Appl. Phys. Lett. 103, 013109 (2013).

${ }^{17}$ R. H. Brown and Q. Twiss, Nature 177, 27 (1956).

${ }^{18}$ D. Renker and E. Lorenz, J. Instrum. 4, P04004 (2009).

${ }^{19}$ B. Lounis, H. Bechtel, D. Gerion, P. Alivisatos, and W. Moerner, Chem. Phys. Lett. 329, 399 (2000).

${ }^{20}$ R. Brouri, A. Beveratos, J.-P. Poizat, and P. Grangier, Opt. Lett. 25, 1294 (2000).

${ }^{21}$ H. Nakajima, H. Kumano, H. Iijima, and I. Suemune, Appl. Phys. Lett. 101, 161107 (2012). 